\title{
Deep Brain Stimulation: Complications and Attempts at Avoiding Them
}

\author{
Roy A.E. Bakay* and Adam P. Smith \\ Rush University Medical Center, Department of Neurosurgery, 1725 W. Harrison Street, Suite 970, Chicago, IL 60612, \\ USA
}

\begin{abstract}
Deep brain stimulation is the most frequent neurosurgical procedure for movement disorders. While this elective procedure is well-tolerated by most patients and very effective, it is not free of complications. These may occur at any step in the DBS procedure, starting with patient selection but may also occur years post-operatively. Most early problems relate to hemorrhage and infection, while most late complications are secondary to hardware failure. This review analyzes the complications that may occur, with emphasis on surgery-related complications for movement disorders and ways to avoid them. While these risks are very low in comparison to other neurosurgical procedures, DBS is still an elective procedure that necessitates extensive care and precision.
\end{abstract}

Keywords: Deep brain stimulation, movement disorders, complications.

\section{DEEP BRAIN STIMULATION: COMPLICATIONS AND ATTEMPTS OF AVOIDING}

Deep brain stimulation (DBS) is the most frequent neurosurgical procedure for movement disorders such as Parkinson's disease (PD), dystonia, and essential tremor (ET). In addition, new applications such as obsessive compulsive disorder, Tourette's syndrome, depression, cluster headache, and epilepsy are increasingly being investigated and may be approved Federal Drug Administration (FDA) in the future. While this elective procedure is well-tolerated by most patients and can be very safely performed, it is not free of complications. These may occur at any step in the DBS procedure, from preoperative patient selection to years postoperatively. This review analyzes the complications that may occur, with emphasis on surgery-related complications for movement disorders, and ways to avoid them.

\section{PRE-OPERATIVE CONSIDERATIONS}

\section{Patient Selection}

This first step in achieving a successful surgery is to operate on the appropriate patient. While patient selection is primarily made by the referring neurologist, it is crucial for the neurosurgeon to concur on the diagnosis, therapeutic approach and viability of the patient as a surgical candidate. It is the surgeon who is ultimately responsible for the operation. In 1999, the Core Assessment Program for Surgical Interventional Therapies in Parkinson's Disease (CAPSITPD) was designed to provide guidelines for patient selection [1]. These guidelines describe the following criteria: 1) the presence of two or more of the cardinal features of PD, one of which must be tremor or bradykinesia; 2) exclusion of patients with atypical parkinsonism; 3 ) disease duration of at least 5 years; and 4) at least a 30\% improvement in the UPDRS-III (motor) score on dopaminergic agents. More

*Address correspondence to this author at the Rush University Medical Center, Department of Neurosurgery, 1725 W. Harrison Street, Suite 970, Chicago, IL 60612, USA; Tel: 312-942-6644; Fax: 312-942-2176;

E-mail: roy_bakay@rush.edu recently, the Movement Disorder Society created a newer version of the UPDRS which integrates the non-motor PD symptoms [2]. The rationale for exclusion of patients with neuropsychological disability or "Parkinson-plus" type syndromes is that these are the least likely to improve and most likely to have complications. Similarly, inclusion of patients with very advanced age, those who do not respond well to Ldopa, those who have severe symptoms in the "on" state are the least likely to improve and most likely to have complications. Furthermore, best results are commonly achieved in tremor, rigidity, and bradykinesia, while "on" gait problems and postural instability poorly responds. While these are not steadfast rules, they are practice-based recommendations. The predominance of non-dopaminergic responsive symptoms is also associated with poor responses. Deviating from these criteria does not guarantee DBS failure, but will commonly lead to less-satisfying results and higher rate of complications.

Patient selection for dystonia and ET is less well defined, as no CAPSIT-like criteria have been formulated. Regarding dystonia, patients with idiopathic primary generalized are considered good surgical candidates. Similarly, genetic forms of dystonia, most commonly involving the DYT1 gene, are considered excellent surgical candidates. Some forms of secondary dystonia respond to DBS, but rarely to the degree observed in primary dystonia patients. In addition, those with appendicular symptoms tend to respond better than those with axial symptoms. For ET, it is commonly observed that postural and distal tremors respond to DBS better than kinetic and proximal tremors, and axial tremor usually requires bilateral lead placement for response [3]. Unfortunately, a subset of ET patients have been shown to lose the effectiveness of their DBS, often requiring additional surgical procedures.

For any DBS surgery, the cognitive status and age of the patient must be assessed, especially in the case of bilateral stimulator placement. Physiological age is more important than chronological age. While debate exists regarding effectiveness of unilateral versus bilateral procedures, it is well 


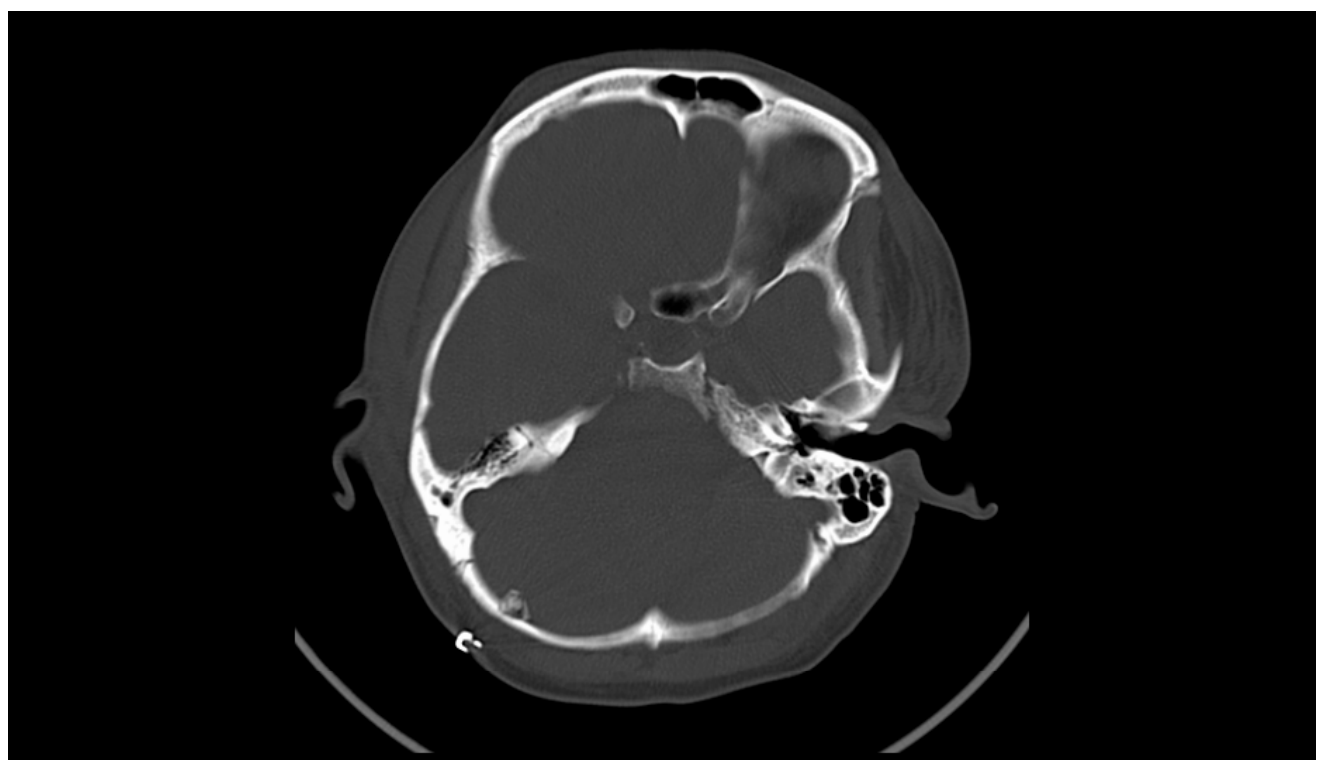

Fig. (1). This is a CT scan which shows injury to the cerebellar hemisphere on the right from over-vigorous tightening of the head pin resulting in skull fracture and cerebellar contusion.

known that the complication rates for post-operative confusion, speech difficulties, and overall cognition are lower with unilateral procedures [4-6]. Specifically, multiple reports describe irreversible dementia after bilateral subthalamic nucleus (STN) DBS in patients whose mental status was borderline pre-operatively [7-9]. In other studies, patients older than 69 years demonstrated higher risk for complications and mental deterioration after STN DBS [10-12]. A recent multicenter randomized study comparing best medical therapy to DBS in advanced PD verified that DBS patients older than 70 years experienced significant improvement but also had higher rates of adverse events [13].

Lastly, all patients undergoing DBS procedures should have proper medical clearance for the procedure and be able to tolerate surgery. Pre-operatively, blood pressure must be well controlled and depression must be treated and stable. Patients should be off any antiplatelet or anticoagulant medications two weeks prior to surgery.

\section{Choosing Location}

While operating on the appropriate patient is crucial, of potentially equal importance is selecting the best operative target for each individual patient. Both the STN and globus pallidus interna (GPi) are the most common targets for PD, and a prospective randomized study failed to show significant efficacy differences between the two locations [14]. While we await a large multicenter randomized trial, most prefer STN because of the ease of targeting and ability to dramatically reduce L-dopa therapy, while others prefer GPi because psychiatric and cognitive decline is less common than in STN. The peduculopontine nucleus has also been studied recently and is an anticipated target to combat postural and gait symptoms in advanced disease [15]. For dystonia and ET, the GPi and ventral intermediate nucleus of the thalamus (VIM), respectively, are most commonly targeted but STN has also been used for these disorders. It remains to be determined if clinical outcome and complication rates are different in these different target sites.

\section{Pre-Operative Imaging}

DBS is a stereotactic procedure requiring the use of preoperative imaging to identify the target. Magnetic resonance imaging (MRI) is most commonly performed, but computed tomography (CT) is sometimes utilized. Increasingly, fusion of both CT and MR images by a computer stereotactic program is used for intra-operative guidance. Applications of frames or bone fiduciaries are rarely subject to minor complication such as skull fracture, loss of registration, etc. (Fig. 1). There appears to be no inherent risks to imaging itself, but poor image quality may drastically affect accuracy of lead placement. With a 1.5 tesla (T) MRI standard in most hospitals and complications attributable to imaging are rare. More critical is potential human error in incorrectly identifying the target. It will be interesting to observe if with the introduction of 3T MRI's whether even higher power MRI's will provide even better target localization and decreased human error by better target elucidation on the pre-operative scans. Image fusion is another potential source for error.

Pre-operative imaging often can provide useful information to help avoid intraoperative complications. Large vessels on the surface and within sulci can be visualized and entry points should be adjusted accordingly. Similarly, it has been shown that targeting error may be attributable to trajectories passing through the ventricle. This can easily be prevented by using current technology to plan trajectories avoiding the lateral ventricle [12]. Lastly, cerebral atrophy in itself is not a contraindication to DBS placement, but severe atrophy may affect targeting due to intraoperative brain shift [16].

\section{OPERATIVE COMPLICATIONS}

Operative complications range from serious lifethreatening issues, such as hemorrhage, venous infarction, and various extra-cerebral problems to more benign entities. Hemodynamic complications are common: hypertension $(59 \%)$, hypotension $(7.9 \%)$, tachycardia $(6.2 \%)$, and brady- 
cardia (18\%) [17]. These potential problems speak to the need for an experienced anesthesia team.

\section{Venous Air Embolism (VAE)}

Intraoperative pulmonary embolism has been reported more commonly in neurosurgical procedures when the patient is in the sitting positioned [18]. Accordingly, DBS procedures, which are frequently performed in the semi-seated position, predispose the patient to similar risk. In contrast, however, this diagnosis is much more difficult in a nonintubated patient without the available end-tidal $\mathrm{CO}_{2}$, precordial Doppler, or transesophageal monitors. Critical findings suggesting VAE are acute onset of intraoperative cough, abrupt change in mental status, hemodynamic instability, and decline in $\mathrm{O}_{2}$ saturation. Placement of a precordial Doppler may supplement these findings to aid the diagnosis. Fortunately, VAE has been reported rarely, although surgeons should be aware of the potential complication $[19,20]$. VAE during DBS procedures will most commonly occur just after burr hole placement. Therefore, quick irrigation of the burr hole copious volumes of saline and sealing with bone wax or fibrin glue should occur as soon as possible. Hypovolemic patients who are awake and breathing spontaneously, typical of many DBS patients, are at high risk due to negative intrathoracic pressures [21]. Incidentally, coughing has been a correlated to instigate the event, and has been thought to induce VAE in sitting position patients via the release of cytokines and complement after coughing-induced vasoconstriction and bronchoconstriction [22]. Performing the burr hole in the supine position and later elevating the patient to the semi-seated position is another means to try and reduce the incidence of VAE.

\section{Infarction}

Venous infarction is also a known complication of DBS, most commonly occurring from transection or coagulation of large draining veins at the site of the burr hole. Placing the burr hole anterior to the coronal suture and as far off midline as possible will decrease the likelihood of a symptomatic complication (Fig. 2). Furthermore, careful attention to vasculature on pre-operative imaging may avoid this complication. The incidence of venous infarction is extremely low, occurring in $0.9 \%$ of patients in one series [23]. Deep subcortical infarcts have also been reported in the literature. Sutton et al., 2004 [24] reported subthalamic nucleus and thalamic ischemia during DBS microelectrode recording. Novak et al., 2006 [25] also reported two cases of ischemia after microelectrode recording of the subthalamic nucleus. The true incidence of deep infarcts is unknown, largely due to unrecognized clinical sequelae or inconsistent follow-up imaging. This may be difficult to distinguish from microlesion effect from microelectrode or lead placement [26]. Deep infarctions during ablative movement disorder procedures have been studied, and a correlation to pre-existing vascular disease has been recognized [27]. The majority of deep infarcts reported after DBS procedures tend to also be associated with small-vessel disease. While some of these cases were identified on post-operative imaging, a few had microelectrode recording silencing noted intraoperatively. As silencing also occurs when the electrode is in edematous or non-cellular areas of the brain, this sign is not diagnostic for infarction, but should raise suspicion especially in high-risk patients. The actual mechanism of deep infarction is unknown, although vasospasm from stimulation or lead com-

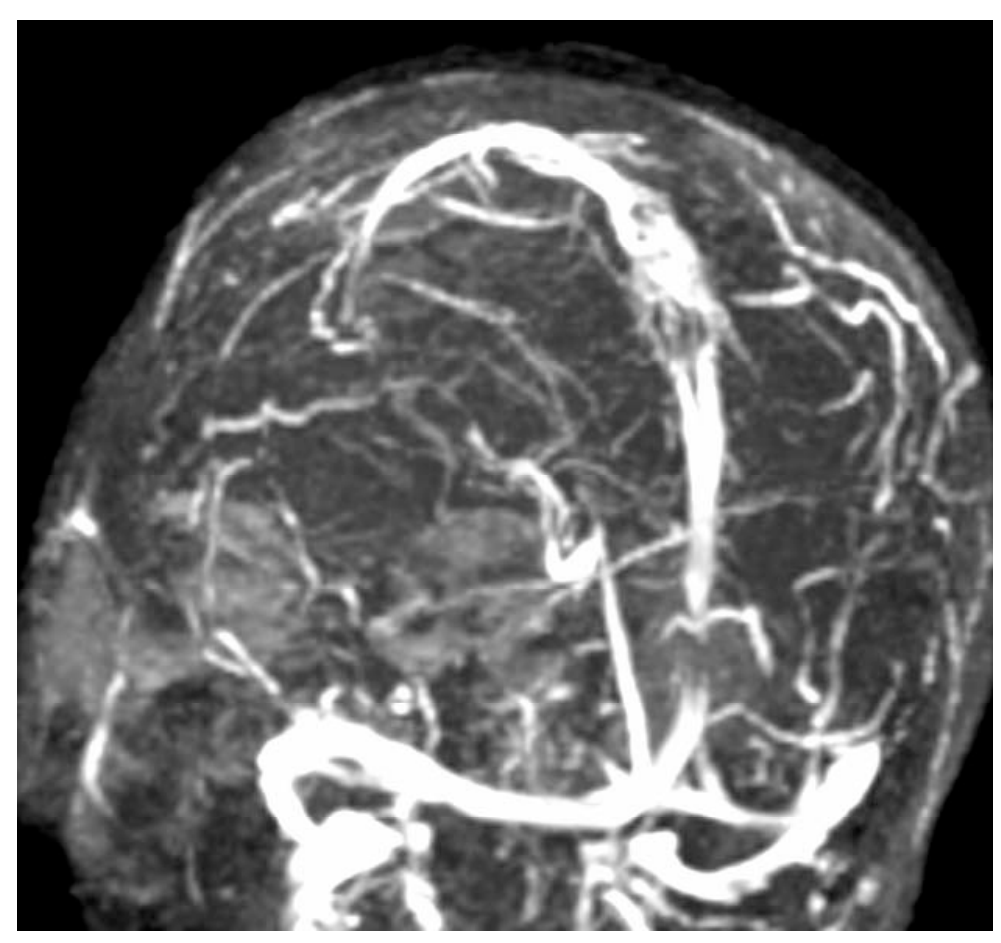

(A)

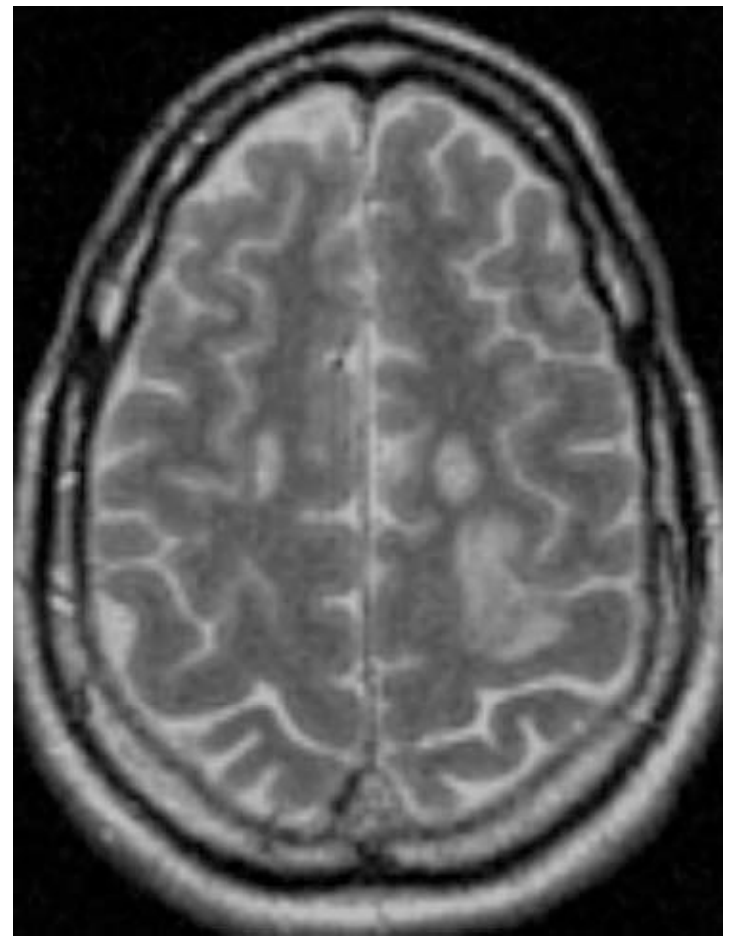

(B)

Fig. (2). This venous phase of an arteriogram (A) demonstrates the lack of venous drainage on the left and a partial occlusion of the sagittal sinus from a blood clot. This is the result of a burr hole too close to the sagittal sinus damaging both the draining vein and the sinus. The result is demonstrated in Fig. (2B) where venous infarction is observed on MRI. 
pression, as well as microvascular vessel rupture are postulated theories.

\section{Seizure}

Intraoperative or post-operative seizures, due to localized ischemia and/or hemorrhagic cortical irritation at the lead entry site, have been previously reported [26, 28-31]. The actual incidence is unknown, but is thought to be similar to the risk of seizure after ventricular shunt placement, which has a rate of less than 5\% during the first year [32]. Recent review reports rates from 0 to $14 \%$ and overall risk approximately $2.4 \%$ [33]. Because of this low risk, and in accordance with ventricular shunt procedures, the risk of medication induced side effects probably outweighs the risk of seizure. Therefore, the prophylactic use of anticonvulsant agents in DBS surgery in not recommended.

\section{Hemorrhage}

Symptomatic subdural hemorrhages do occur, but are very uncommon $(<1 \%)$ [34]. Symptomatic intracerebral hemorrhage from all stereotactic surgery, including ablation, has been reported to occur between 1-5\% [35-40]. The incidence in DBS procedures is low, ranging from $0.7-3.3 \%$ per lead with a permanent morbidity of $0.6-0.8 \%$ [28, 41-43]. The potential for hemorrhage increases with age and preexisting hypertension but this has not always been confirmed $[11,40,42,44,45]$. The increased risk of hemorrhage occurs more frequently in the hypertensive patient $(10.7 \%$ in hypertensive patients compared to $0.91 \%$ in normotensive patients) in one study [45]. We believe vigilant intraoperative blood pressure monitoring to keep the mean pressure at or below $90 \mathrm{~mm} \mathrm{Hg}$ is helpful in reducing the risk of this complication. Furthermore, careful pre-operative planning to avoid all potential vessels crossing the proposed trajectory may be crucial to avoidance of this complication. Passage through the ventricle may increase the risk of hemorrhage [42]. Hemorrhage in the pocket for the impulse pulse generator (IPG) is a rare problem and usually does not require surgery unless the suture line is at risk.

\section{Microelectrode Recording (MER)}

Most functional surgeons use electrophysiology to determine the final target location. Thus, microelectrode recordings (MER) and/or macrostimulation is used to "define" the target nucleus and identify the optimal location for final lead placement. Due to the additional data attained with MER, it is used in most centers. Multiple studies verify the safety of MER [13, 28, 30, 39, 44, 46-48]. Some reviews suggest a trend toward an increased incidence of hemorrhage when MER is used and especially with multiple MER passes $[30,45,48]$ but others have not found such a trend [41, 42]. However, this is in comparison to the overall risk of hemorrhage in stereotactic procedures for movement disorders which ranges from 1-3\%, so no definitive conclusions can be established (Fig. 3). Furthermore, the type of microelectrode may make a difference [44]. Although its usefulness has been questioned for routine use, due to the potential increased risk of hemorrhage with multiple parenchymal passes and potential increased incidence of infection due to prolonged surgical time, there is no statistically valid evidence of increased hemorrhage rates with MER or secondary to multiple passes. Most neurosurgeons use MER in their
DBS procedures, believing the additional data outweighs the potential risk of hemorrhage.

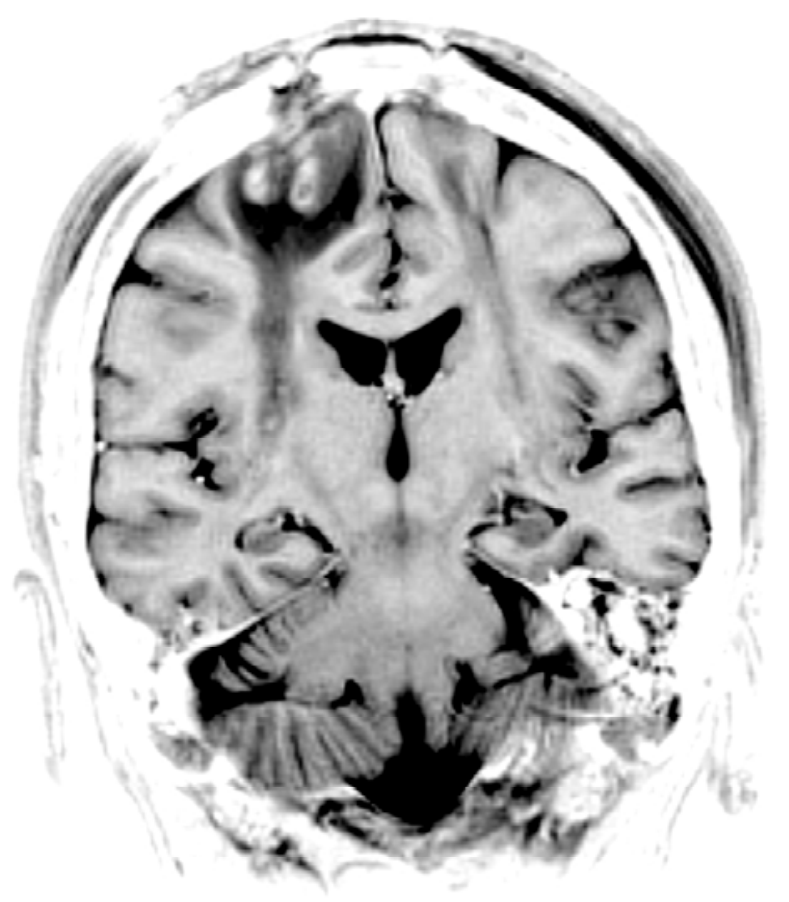

Fig. (3). This MR image demonstrates the edema surrounding a tract seen on the left. In addition, there are hemorrhagic changes observed on the right. This is not from MER or DBS leads but actually from transplantation catheters and demonstrates that simply passage of tubes through the brain has risks.

\section{Pulmonary Embolism}

Pulmonary embolism occurring in the early postoperative setting after neurosurgical procedures ranges from 0.4 to $4.9 \%$, with mortality from such ranging from 8.6 to $59.4 \%$ [35]. This complication, including death from pulmonary embolism, has been reported following DBS procedures in scattered reports $[12,17,31,49]$. Standard prophylaxis through the use of compressive boots or stockings and early mobilization with rehabilitation therapy are believed essential to help avoid this complication. Prophylactic dose subcutaneous anticoagulants may be helpful in patients with prolonged immobility.

\section{POST-OPERATIVE COMPLICATIONS}

\section{Device-Related Complications}

Device-related complications include infection and/or erosion, electrode disconnection or fracture, migration, and IPG malfunction. The more common complications will be discussed but pain, cerebrospinal fluid (CSF) leak, ballism, eye lid apraxia, hepatic failure, respiratory failure, foreign body reaction, pulmonary embolus, etc. are very uncommon $(<1 \%)$ and will not be discussed in detail.

\section{Infection}

The DBS hardware is a foreign body and is therefore prone to infection. There is a $4-12.2 \%$ risk per patient or 1.5 $9.7 \%$ risk per lead of perioperative infection $[13,40,43,46$, 50-53]. The most common organism cited in the literature is Staphylococcus aureus. Infection incidence reporting, how- 


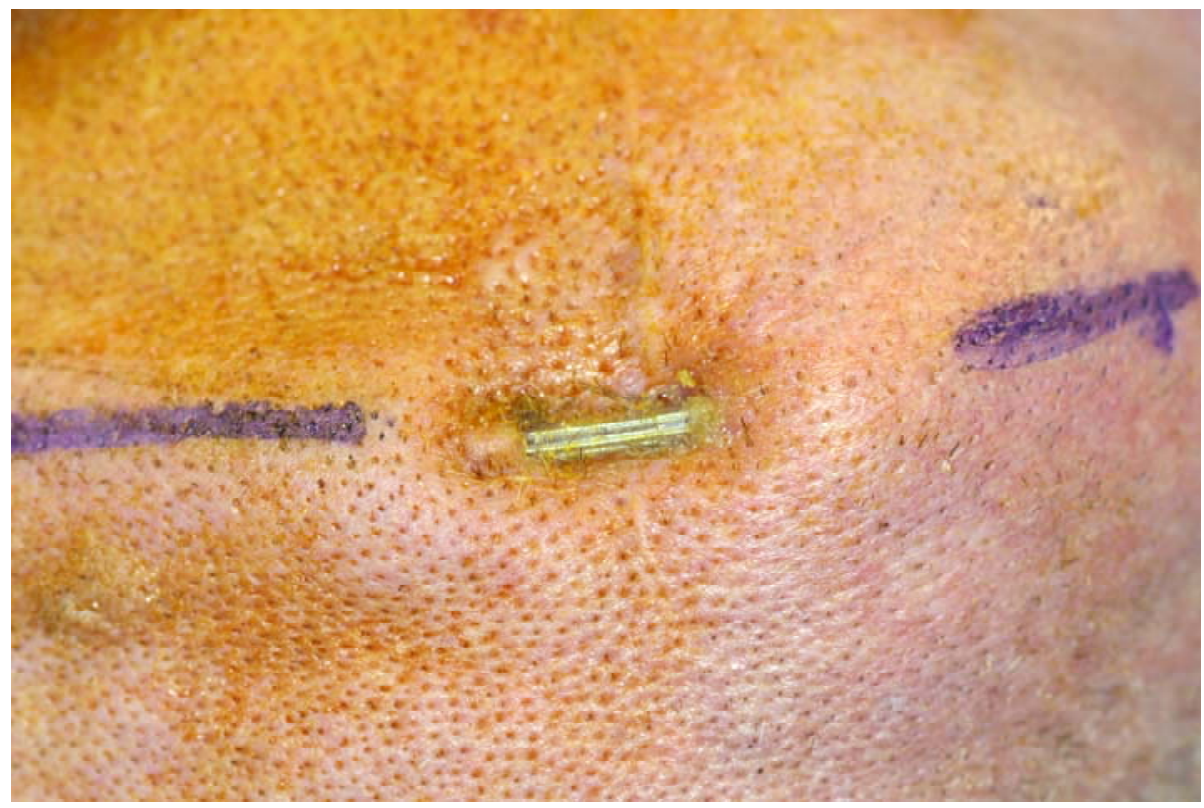

Fig. (4). This is an unusual erosion where the extension lead eroded through the skin. Simple removal of the extension lead and replacement was performed without loss of function.

ever, is complicated in that different authors often use vastly different definitions for infections. Infections can grossly be divided into superficial versus deep, and early versus late. Superficial infections appear to be the most common. One series reported a $5.7 \%$ skin infection rate, with $1.5 \%$ occurring within the first month and $6.1 \%$ thereafter. Mean time to first signs of infection was 3.7 months amongst 15 of the 262 patients who experienced infection [43]. This highlights that even superficial infections often occur later, after the traditional perioperative time. At our institution we record an overall $2.8 \%$ infection rate, with $1.4 \%$ being superficial skin infections. To combat all types of infection, routine use of prophylactic antibiotics perioperatively is recommended. We copiously irrigate the operative field with vancomycin (or bacitracin if allergic) irrigation throughout the procedure and before approximating the skin at the end of the procedure. We use an intravenous cephalosporin (or cleocin if allergic) perioperatively and for twenty-four hours after the procedure. We then continue with an oral antibiotic for up to 5 days postoperatively. Additionally, we cover the Stimloc system with pericranium and bury the lead and connector below the temporalis fascia to further deter wound breakdown and infection.

Once infected, antibiotic therapy alone for nonsuperficial infections involving the hardware is of limited utility. Surgical debridement, with or without hardware removal, is often warranted. The degree and location of infection dictates if and how much of the DBS system may need to be removed and later replaced. At our institution, we treat all deep infections with surgical debridement and intravenous antibiotics. We have a $60 \%$ success rate in salvaging leads with debridement and antibiotics without hardware removal. Failures occurred with gram negative organisms and co-morbidities such as diabetes. In other series, partial or complete hardware removal is reported as low as $4.6 \%$ [43, 54]. Others never have had to remove an infected lead. Commonly, attempts at only partial hardware removal of the
IPG or lead extender are preferable and often sufficient compared to the complete removal.

The device, even in low profile models, is prominent under the scalp, neck, or subclavicular tissues. This unfortunately predisposes to erosion through the skin and risk for infection. Currently, the most common site of erosion is at the connection site between the DBS lead and extension lead. Of interest, in one series involving few deep hardwarerelated infections associated with erosions, the majority (68\%) arose from the IPG [54]. However, if erosion is present, the site of erosion is almost always infected (Fig. 4). The combined deep infection and erosion rates have been reported between $1.5-15.2 \%[9,13,28,30,31,40,43,46$, $50,51,55]$. Including erosions, the infection rate at our institution is $3.4 \%$. It is important to note, however, that these two entities do not always occur in conjunction, but requires breakdown of the skin covering the IPG when they do. Infections can occur late after surgery without erosion, and erosion can occur early after surgery without infection. In the case of erosion with infection, surgical debridement is crucial and hardware often needs to be removed since antibiotics alone will unlikely suffice. In the event of erosion with negative cultures, it is at the surgeon's discretion for management. In one series, the reported rate of erosion alone, without infection, was $1.5 \%$ [50]. Whatever the cause, the erosion must be dealt with if successful treatment is to be accomplished. The use of low-profile hardware placed deep (IPGs implanted within the muscle of thin patients) and away from the incision lines theoretically should decrease the incidence of erosion. There is also the option of IPG placement in the abdomen, but that may increase the risk of migration and other complications.

Although not statistically significant, the Kinetra doublechannel device appears to have an increased infection rate compared to either the Soletra or Itrel II single-channel devices, most likely due to the smaller size of the latter two 
[54]. The subcutaneous pocket created in the subclavicular tissue to house the IPG may be too large or disrupt lymphatics. Within this pocket, a seroma may form with a potential to develop incisional breakdown or subsequent infection exists. Although most will spontaneously resolve, a seroma may be treated with antibiotic prophylaxis and occasionally needs to be aspirated to relieve pressure on the suture line. The greatest concern is to salvage the DBS lead and we will always try to preserve it. Actual intracranial infection is rare even in the case of DBS lead infection. After removing hardware, most authors wait at least two months before reimplantation [54]. We wait for normalization of the erythrocyte sedimentation rate and C-reactive protein values.

Certain surgical techniques such as non-linear incisions have been attempted by numerous authors to lower infection rates. At our institution we use non-linear incisions, meticulously avoid placing hardware directly under the suture line. Other factors may include use of prophylactic antibiotics, limited handling of the hardware, smaller incisions, meticulous surgical technique, shaving with clippers rather than razors, use of alcohol, betadine and chlorhexidine skin prep, irrigating with antibiotic solutions, placing permanent anchors only in deep tissue, and placing the IPG underneath muscle fascia rather than in the fat. Operative time is probably not a factor [54].

\section{Lead Fracture, Migration, and Misplacement}

Lead fracture or disconnection is another common complication of DBS (Fig. 5). The rate of lead fracture has been reported between $2.0-9.9 \%$ per patient, or $\sim 1.8 \%$ per lead implanted $[50,55]$. Lead fracture may produce a short or open circuit, which has been reported to occur in $0.9-9.9 \%$ of patients $[28,36,37,55]$. In the reported literature, few studies categorize short circuit or open circuit separate from lead fracture (Fig. 6). Lead fracture has been correlated with connecting the DBS lead to the extension lead in the upper neck area, below the mastoid [50,56-58]. As high as $42 \%$ of lead fractures have been attributed to the lead connector sitting below or on the mastoid [58]. Neck movements may increase pressure on the connector site leading to fracture. It has been reported that fracture incidence can be minimized by securing this connection higher on the calvarium [56]. Of interest, some authors note that the majority of lead fractures occur in ET patients, probably due to increased neck movement with tremor and to increased patient mobility compared to matched patients with PD or Dystonia [50]. Whatever the cause, lead fracture necessitates re-operation with removal of the damaged lead and replacement (Fig. 7). It is most commonly reported 6-24 months after initial operation [51, 55, 59]. As the very long-term follow-up data of DBS is attained, this complication may increase.

DBS lead migration has been reported in $1.5-6.3 \%$ of patients, or $\sim 4.4 \%$ of leads implanted [34, 40, 53, 59]. In one small series, the reported incidence was as high as $14.2 \%$ but was performed with totally different equipment [37]. Migration can occur immediately and the patient never benefited from the surgery but most commonly it is detected between 6 months to 3 years after initial surgery, when the patient notes

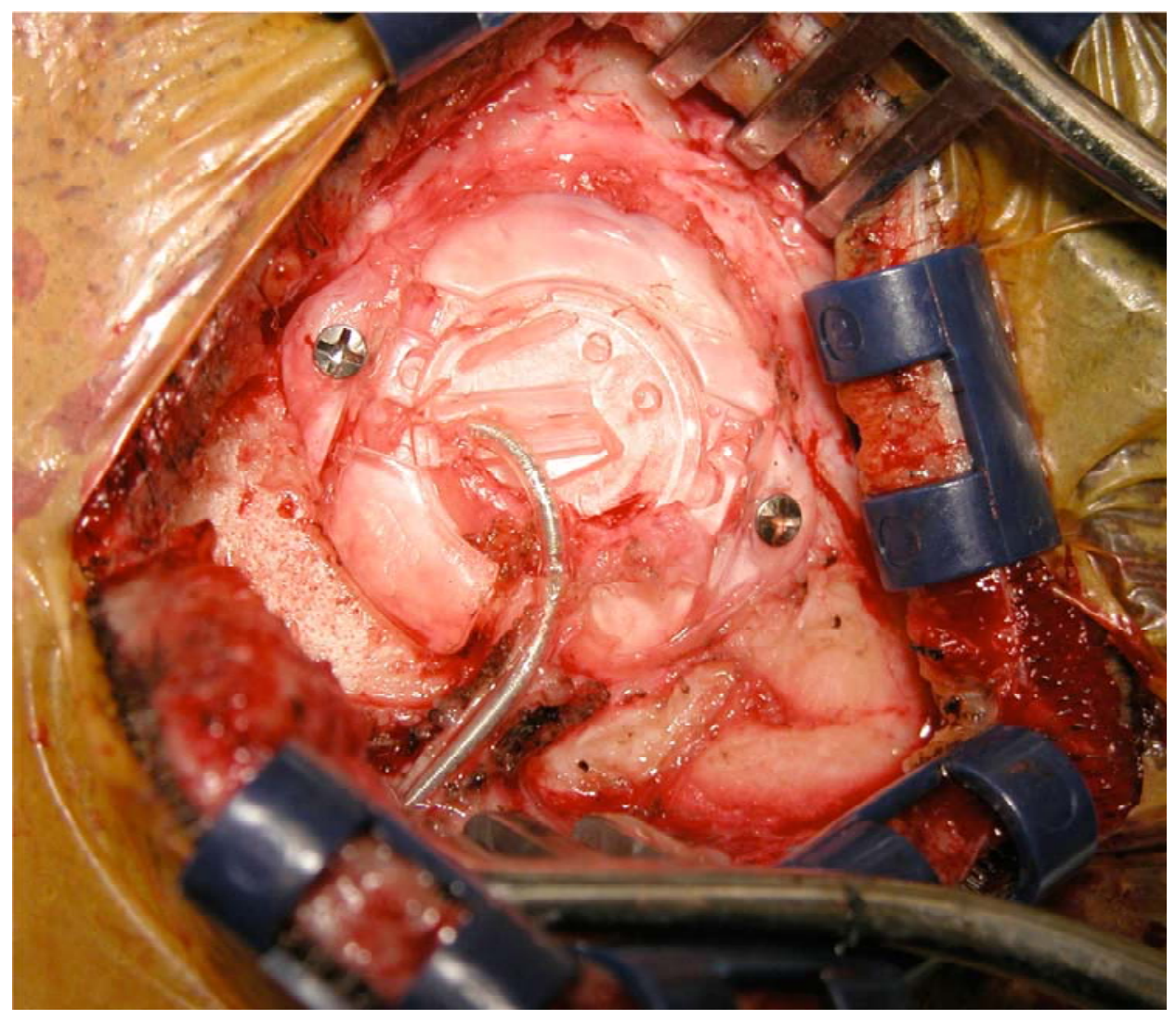

Fig. (5). This patient's DBS never worked. The picture shows a fracture in the DBS lead from improper placement of the lead in the Stimloc system. Rather than being secured in the groove, the lead is posterior to the groove and was compressed by the cap, resulting in a fracture at the edge of the plastic. 


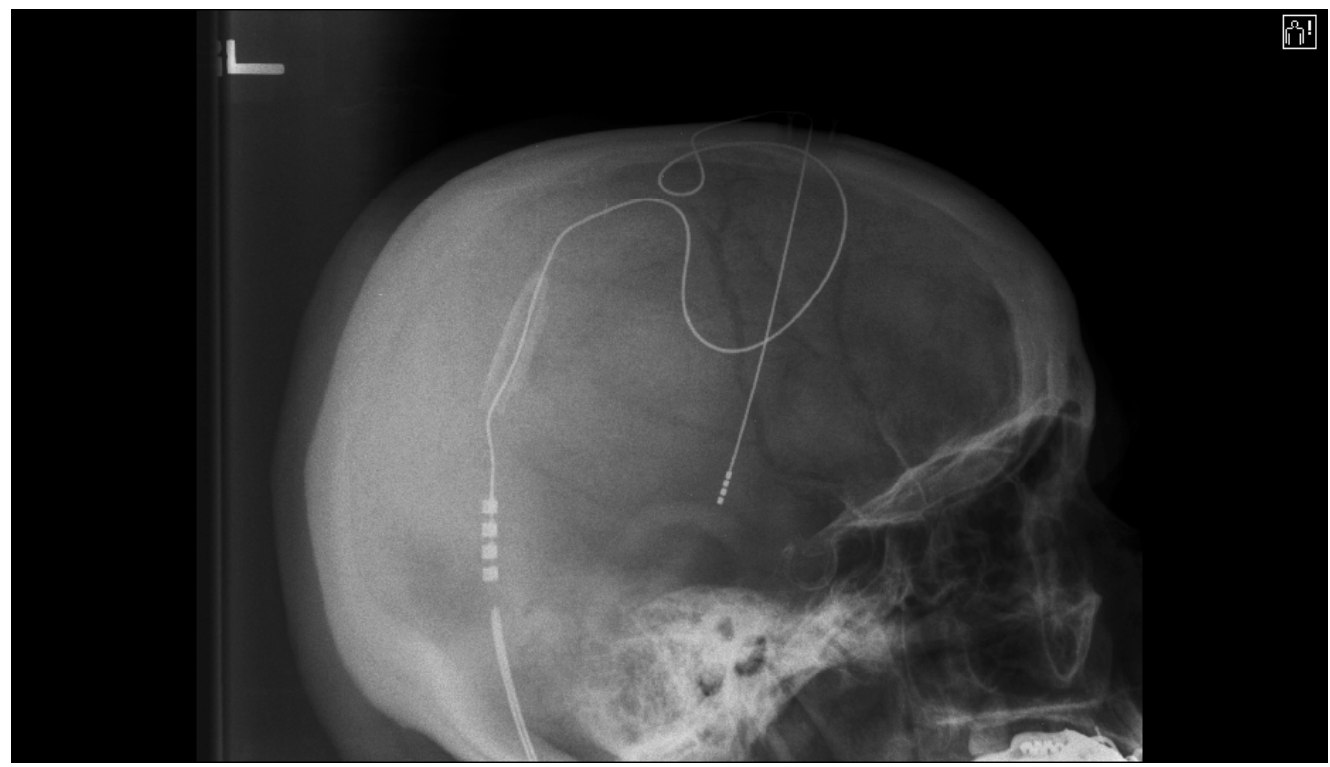

Fig. (6). There is a most unusual disruption of the boot and the connector seen in this x-ray. The result is that fluid is in contact with all of the electrical contacts and as a result, function of the lead is compromised.
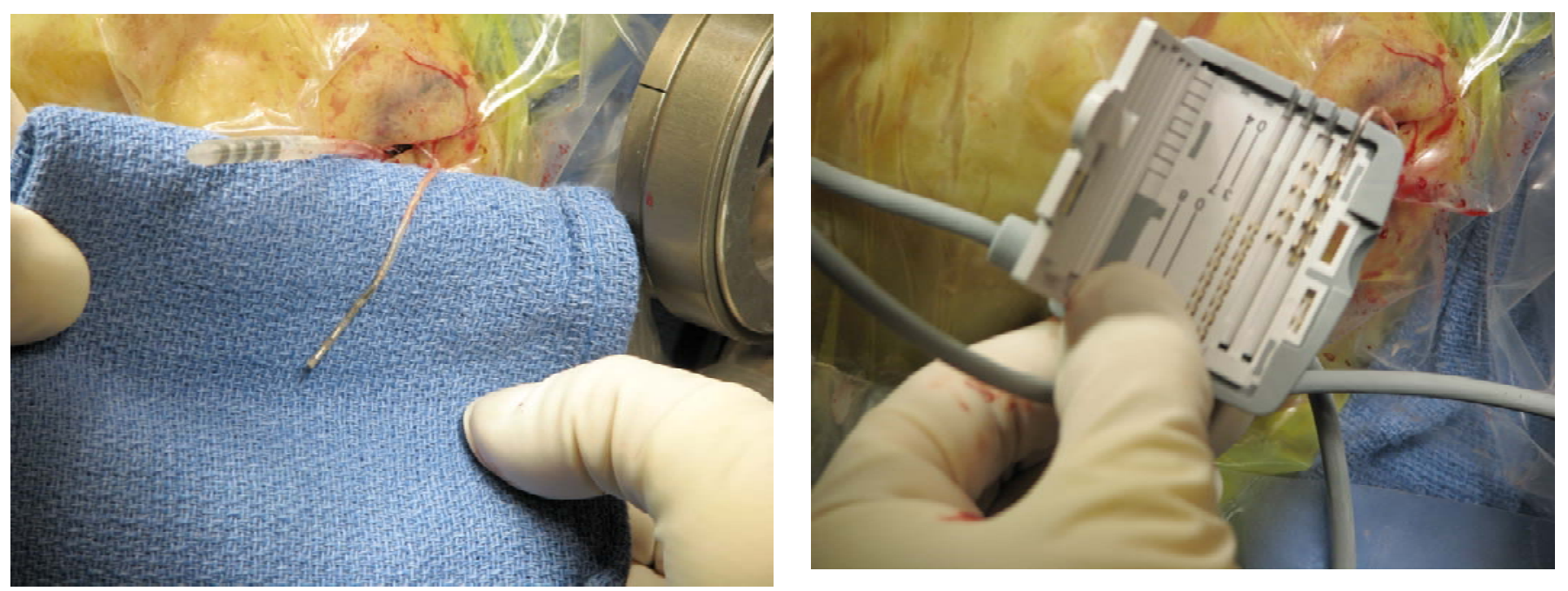

Fig. (7). There are times when it is not clear whether the fracture is in the extension lead or the DBS lead. Although there is discoloration of this DBS lead in A, when tested as seen in B, the difficulty rested not with the intracranial portion but with the extension lead which was replaced and function restored.

decline in function [59]. If this occurs, the patient must return to surgery for correction of DBS lead placement.

The etiology of lead migration has often been attributed to numerous factors, whether inadequate securing of the lead to the calvarium, patient-induced from involuntary movements, or improper location of the connector in the neck. With the advent of the Stimloc system by Medtronic, the lead is better secured to the burr hole, thus helping prevent lead migration from target even if the distal lead is disrupted. In similar manner, securing the DBS lead and extension lead connection above the mastoid avoids pressure below, leading to a lower likelihood of connection fracture and migration. At our institution, we place the connection below the temporalis fascia and muscle behind the ear. We secure it with suture to the fascia and cover it with as many layers as possible to avoid lead fracture, migration, erosion or infection.
Lead migration usually is a late complication, but may also occur intraoperatively often in the process of securing the DBS lead in place (Fig. 8). This intra-operative migration was reported in one series to occur as frequently as $1.5 \%$ of the time [50]. In this scenario, migration is easily detected either by intraoperative fluoroscopic imaging where it is corrected at the time, or later by post-operative imaging. In the latter case, the patient will need to return to the operating room to correct the complication. For this reason, we routinely utilize intraoperative fluoroscopy after securing the lead in place to identify any such migration and allow correction at the time of initial surgery.

Overall hardware complications occur in 7.8 to $32.9 \%$ of patients from misplaced lead, loss of effect, lack of efficacy, lead fracture, lead migration or infection [34, 51, 52, 53, 55]. An extensive review suggests a better estimate is approxi- 


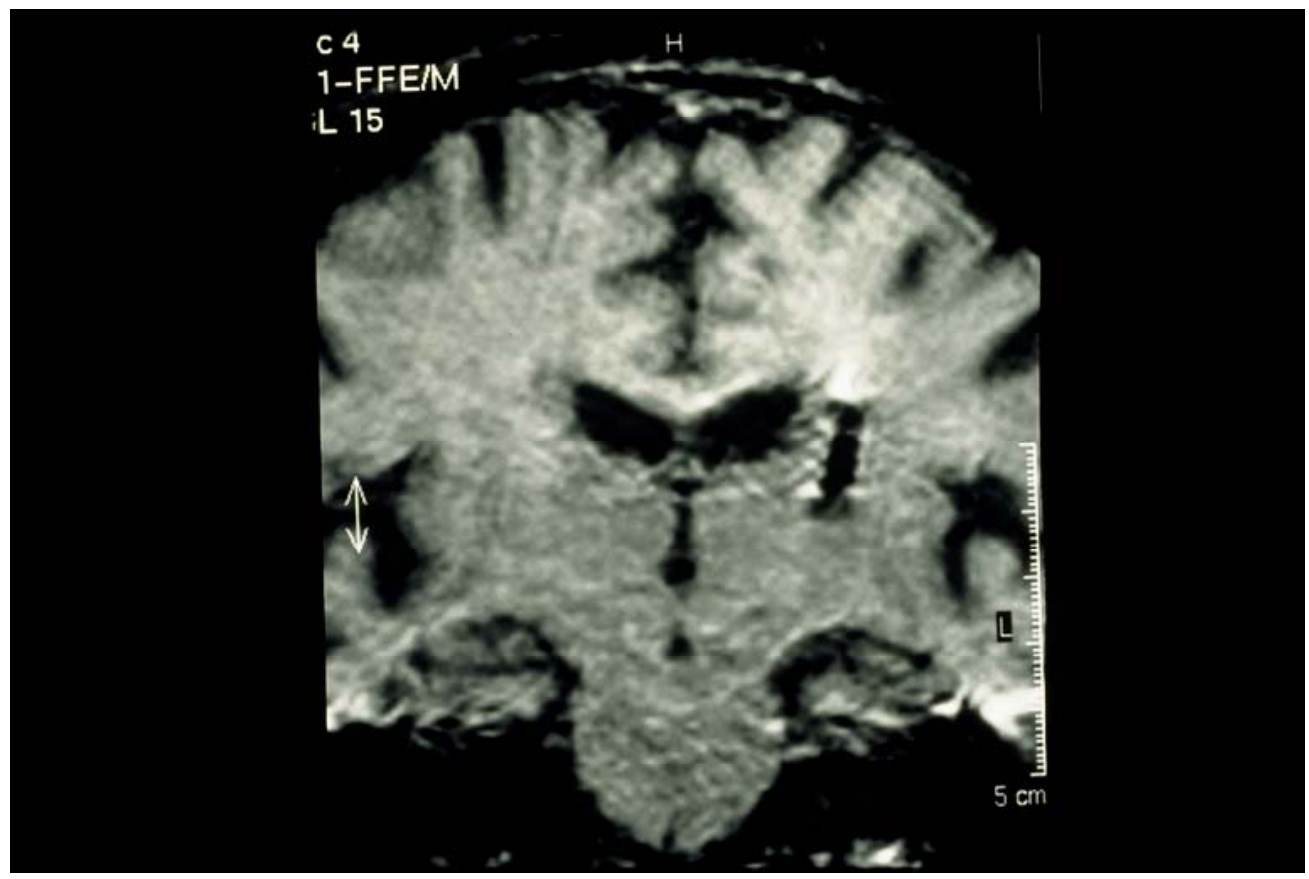

Fig. (8). The DBS lead here has apparently migrated away from the target and the DBS was totally non-functional. The patient was told that they were simply "one of those patients that did not respond to therapy". However, revision of the lead produced excellent symptomatic improvement.

mately $8 \%$ per lead-year [40]. There are many reasons for "failed DBS" but the correction of medications, stimulation parameters, or lead placement in expert hands can correct up to $51 \%$ of these "failures" [60]. Tissue toxicity does not appear to be a problem. Histopathlogical studies have demonstrated that DBS leads cause minimal damage to the adjacent tissue, with only mild gliosis around the implanted lead track [61]. Despite this, long-term data is still being accrued.

\section{Stimulation}

Stimulation-related complications also occur, most dependent on the stimulation target and the actual location of the lead in the given nucleus [40]. Reported symptoms are eyelid apraxia, dyskinesia, dysarthria, paresthesia, diplopia, blepharospasm, and a variety of symptoms from corticospinal tract stimulation. These are probably the most common reported complications by patients and, in part, may be predicted by interrogative testing. Most of these effects are reversible through adjustment of the stimulation parameters, although the effects of stimulation on the movement disorder symptoms may subsequently diminish. Stimulation of STN usually manifests as increased dyskinesia, dysarthria, paresthesia, blepharospasm, confusion and depression, and mental status changes [40, 46, 50]. It is the increased mental status changes that potentially make STN less attractive than GPi as a target. Furthermore, it appears that patient age and preoperative cognition are more important in predicting postoperative cognitive decline in STN than GPi or Vim DBS. Stimulation of GPi may produce visual disturbance, paresthesia, gait and speech problems, as well as confusion and depression. Lastly, stimulation of Vim may produce paresthesias, dysarthria, dizziness, gait and balance problems, muscle cramps, and decreased proprioception and fine motor control. Weight gain is common but may be more related to decreased symptoms rather than a direct stimulation effect.

\section{Cognitive and Neuropsychiatric Problems}

The effects of DBS on the cognitive and neuropsychiatric symptoms transiently are common but any long term effects are controversial $[40,62,63]$. The neuropsychiatric symptoms are primarily transient, treatable, and potentially preventable [62]. Whether preoperative psychiatric symptoms improve or worsen following surgery is also not clearly established. As part of the selection process for surgery, patients are chosen for their lack of overt dementia or other active disabling psychiatric symptomatology rendering analysis difficult. In a randomized study that compared DBS with the best medical treatment, 123 patients had neuropsychological and psychiatric examinations to assess the changes between baseline and 6 months post-operative [63] After 6 months, impairments were seen in executive function, verbal fluency, and Stroop naming time irrespective of the improvement in quality of life suggesting that STN DBS does not reduce overall cognition or affectivity while there is a selective decrease in frontal cognitive functions. The authors concluded that STN DBS is safe with respect to neuropsychological and psychiatric effects in carefully selected patients during a 6-month follow-up period. Observed suicide rates in the first postoperative year are higher than expected [62]. Postoperative depression, unmarried status, younger patients and a previous history of impulse control disorders or compulsive medication use are independent associated factors for attempted and completed suicide risk. Postoperative depression remained a significant factor associated with attempted and completed suicides and is one of the most important potentially preventable risks for mortality following STN DBS for PD. Other factors rarely considered are social and job-related factors, development of dementia, general medical issues, and lifestyle changes. Site of stimulator may also be significant as more cognitive and psychological problems are reported in STN than GPi [40, 62]. 


\section{IPG Malfunction}

External interference is a common problem with stimulators. This often causes the IPG "switching off", and can result in acute clinical deterioration. The reported incidence of frequent external interference is $1.5 \%$ [50]. This previously was a common problem most often observed with the Itrel II neurostimulator, but has decreased in incidence with the advent of the Kinetra model, which shields against external magnetic interference. There was one recall of the Kinetra for mechanical problems, but this has since been corrected. Most occurrences of IPG failure are easily detected and corrected without morbidity. There are a few reports, however, of external interference resulting in severe morbidity either from the stimulator being turned off with acute clinical deterioration or the stimulator actually increasing amplitude leading to significant thermal lesioning [56, 64, 65]. We do not consider pure end of life battery failure a complication of DBS, but instead a routine accompaniment to the procedure.

\section{Post-Operative Imaging}

Post-operative imaging is standard practice to verify location of DBS leads and to assess possible surgical complications. MR imaging is the most popular of imaging options. Unfortunately, the powerful electromagnetic fields emitted by MR may induce serious injury to brain tissue by heating at the electrodes. In the reported literature, only two cases exist in which a neurologic event (one to the brain and one to the lumbar spine) in a DBS patient was induced by MR [66, 67]. Despite the risk, multiple studies have confirmed the safety of MRI to be performed above the neck for DBS patients [23, 68, 69]. Medtronic recommends limiting the exam to the head with a specific absorption rate (SAR) to 0.1 $\mathrm{W} / \mathrm{kg}$, although studies commonly report $0.4 \mathrm{~W} / \mathrm{kg}$ SAR as safe. A SAR of up to $3.0 \mathrm{~W} / \mathrm{kg}$ has been reported [68].

SAR is defined differently by different MR manufacturers, making standardization problematic. In a recent study of 42 centers using MRI for DBS, only one center reported a complication and this occurred without any long-term neurologic deficit. Most centers reported that they turned the stimulator "off", set the amplitude of the IPG to $0 \mathrm{~V}$, checked the impedance and current, and only half obtained consent [69]. While standard 1.5T MRI has been proven safe for use in DBS patients, no studies yet exist regarding $3 \mathrm{~T}$ or even more powerful MR imaging.

In accordance with maintaining safety and in following MRI recommendations in DBS patients, any concomitant spine issues should be evaluated and treated before DBS placement. All appropriate imaging studies should be addressed because after the DBS is placed, MRI below the neck is contraindicated.

\section{Death}

Death from DBS surgery is a rare occurrence. Some report an incidence of $0.4-1.8 \%[12,31,37]$. Reviews put perioperative death at about $0.4 \%[40,70]$. Causes of death are intracranial hemorrhage, pneumonia, pulmonary embolism, and suicide. The occurrence of suicide attempts is $0.5 \%$ and is possibly a preventable cause of death [62].

\section{CONCLUSION}

While DBS is now a common and beneficial procedure for patients with movement disorders, it is associated with significant, albeit uncommon, complications. These risks are extremely low in comparison to other neurosurgical procedures. Despite this, most of the complications are associated with hardware failure. As technology improves it is imperative that neurosurgeons communicate with manufacturers to improve the safety of the devices. In adjunct, neurosurgeons must continue to refine their own surgical technique to minimize complications. Despite its benefits, DBS is still an elective procedure that necessitates minimal risk to the patient.

\section{REFERENCES}

[1] Defer GL, Widner H, Marie RM, Remy P, Levivier M. Core assessment program for surgical interventional therapies in Parkinson's disease (CAPSIT-PD). Mov Disord 1999; 14: 572-84.

[2] Goetz CG, Tilley BC, Shaftman SR, et al. Movement disorder society-sponsored revision of the unified parkinson's disease rating scale (MDS-UPDRS): scale presentation and clinimetric testing results. Mov Disord 2008; 23(15): 2129-70.

[3] Putzke JD, Uitti RJ, Obwegeser AA, Wszolek ZK, Wharen RE. Bilateral thalamic deep brain stimulation: midline tremor control. J Neurol Neurosurg Psychiatry 2005; 76: 684-90.

[4] Alberts JL, Hass CJ, Vitek JL, Okun MS. Are two leads always better than one? an emerging case for unilateral subthalamic deep brain stimulation in Parkinson's disease. Exp Neurol 2008; 214: $1-5$.

[5] Alberts JL, Voelcker-Rehage C, Hallahan K, Vitek M, Barnzai R, Vitek JL. Bilateral subthalamic stimulation impairs cognitivemotor performance in Parkinson's disease patients. Brain 2008, 131: 3348-60.

[6] Slowinski JL, Putzke JD, Uitti RJ, et al. Unilateral deep brain stimulation of the subthalamic nucleus for Parkinson disease. J Neurosurg 2007; 106(4): 626-32.

[7] Foncke E, van der Linden C, Vandewalle V, Colle H, Caemaert J. Chronic bilateral stimulation of the subthalamic nucleus (STN) in 15 patients with advanced idiopathic Parkinson's disease previously treated with unilateral internal globus pallidus (GPi) stimulation. Neurology 2001; 56(Suppl 3): A272.

[8] Hariz MI, Johansson F, Shamsgovara P, Johansson E, Hariz GM, Fagerlund M. Bilateral subthalamic nucleus stimulation in a Parkinsonian patient with preoperative deficits in speech and cognition: persisting improvement in mobility but increased dependency: a case study. Mov Disord 2000; 15: 136-9.

[9] Limousin P, Krack P, Pollak P, et al. Electrical stimulation of the subthalamic nucleus in advanced Parkinson's disease. N Engl J Med 1998; 339: 1105-11.

[10] Saint-Cyr JA, Trepanier LL. Neuropsychologic assessment of patients for movement disorder surgery. Mov Disord 2000; 15: 771-83.

[11] Vesper J, Haak S, Ostertag C, et al. Subthalamic nucleus deep brain stimulation in elderly patients - analysis of outcome and complications. BMC Neurol 2007; $7: 7$.

[12] Voges J, Hilker R, Rotzel K, et al. Thirty days complication rate following surgery performed for deep-brain-stimulation. Mov Disord 2007; 22(10): 1486-9.

[13] Weaver FM, Follett K, Stern M, et al. Bilateral deep brain simulation versus best medical therapy for patients with advanced Parkinson disease. JAMA 2009; 301(1): 63-73.

[14] Follett KA, Weaver FM, Stern M, et al. Pallidal versus subthalamic deep-brain stimulation for Parkinson's disease. N Engl J Med 2010; 362(22): 2077-91.

[15] Hamani C, Stone S, Laxton A, Lozano AM. The pedunculopontine nucleus and movement disorders: anatomy and the role for deep brain stimulation. Parkinsonism Relat Disord 2007; 13: S276-80.

[16] Miyagi Y, Shima F, Sasaki T. Brain shift: an error factor during implantation of deep brain stimulation electrodes. J Neurosurg 2007; 107: 989-97.

[17] Santos P, Valero R, Arquis MJ, et al. Preoperative adverse events during stereotactic microelectrode-guided deep brain surgery in 
Parkinson's disease. Rev Esp Anestesiol Reanim 2004; 51(9): 52330 .

[18] Balki M, Manninen DH, McGuire GP, El-Beheiry H, Bernstein M. Venous air embolism during awake craniotomy in a supine patient. Can J Anesth 2003; 50: 835-8.

[19] Deogaonkar A, Avitsian R, Henderson JM, Schubert A. Venous air embolism during deep brain stimulation surgery in an awake supine patient. Sterotact Funct Neurosurg 2005; 83: 32-5.

[20] Moitra V, Permut T, Penn R, Roth S. Venous air embolism in an awake patient undergoing placement of deep brain stimulators. $\mathrm{J}$ Neurosurg Anesthesiol 2004; 16: 321.

[21] Suarez S, Ornaque I, Fabregas N, Valero R, Carrero E. Venous air embolism during Parkinson surgery in patients with spontaneous ventilation. Anesth Analg 1999; 88: 793-4.

[22] Smith DS, Osborn I. Posterior fossa: anesthetic considerations. In: Cottrell JE, Smith DS, Eds. Anesthesia and neurosurgery, $4^{\text {th }}$ ed. St. Louis: Mosby 2001; pp. 335-51.

[23] Tronnier VM, Staubert A, Hahnel S, Sarem-Aslani A. Magnetic resonance imaging with implanted neurostimulators: an in vitro and in vivo study. Neurosurgery 1999; 44: 118-26.

[24] Sutton JP. Deep brain stimulation (DBS) of the subthalamic nucleus (STN) and thalamic ischemia: a report of two cases. Mov Disord 2004; 19(Suppl 9): S324.

[25] Novak KE, Nenonene EK, Bernstein LP, et al. Two cases of ischemia associated with subthalamic nucleus stimulator implantation for advanced parkinson's disease. Mov Disord 2006; 21(9): 1477-83.

[26] Mann JM, Foote KD, Garvan CW, et al. Brain penetration effects of microelectrodes and DBS leads in STN or GPi. J Neurol Neurosurg Psychiatry 2009; 80(7): 794-7.

[27] Lim JY, DeSalles AA, Bronstein J, Masterman DL, Saver JL. Delayed internal capsule infarctions following radiofrequency pallidotomy: report of three cases. J Neurosurg 1997; 87: 955-60.

[28] Benabid A, Chabardes S, Mitrofanis J, Pollak P. Deep brain stimulation of the subthalamic nucleus for the treatment of Parkinson's disease. Lancet Neurol 2009; 8(1): 67-81.

[29] Seijo FJ, Alvarez-Vega MA, Gutierrez JC, et al. Complications in subthalamic nucleus stimulation surgery for treatment of Parkinson's disease. Review of 272 procedures. Acta Neurochir (Wien) 2007; 149(9): 867-75; discussion 876.

[30] The Deep-Brain Stimulation for Parkinson's Disease Study Group. Deep-brain stimulation of the subthalamic nucleus or the pars interna of the globus pallidus in parkinson's disease. N Engl J Med 2001; 345: 956-63.

[31] Umemura A, Jaqqi JL, Hurtig HI, et al. Deep brain stimulation for movement disorders: morbidity and mortality in 109 patients. J Neurosurg 2003; 98(4): 779-84.

[32] Dan NG, Wade MJ. The incidence of epilepsy after ventricular shunting procedures. J Neurosurg 1986; 65: 19-21.

[33] Coley E, Farhadi R, Lewis S, Whittle IR. The incidence of seizures following Deep Brain Stimulating electrode implantation for movement disorders, pain and psychiatric conditions. Br J Neurosurg 2009; 23(2): 179-83.

[34] Kenney C, Simpson R, Hunter C, et al. Short-term and long-term safety of deep brain stimulation in the treatment of movement disorders. J Neurosug 2007; 106(4): 621-5.

[35] Jankovic J, Cardoso F, Grossman RG, Hamilton WJ. Outcome after stereotactic thalamotomy for parkinsonian, essential and other types of tremor. Neurosurgery 1995; 37: 680-7.

[36] Kumar K, Toth C, Nath R. Deep brain stimulation for intractable pain: a 15-year experience. Neurosurgery 1997; 40: 736-46.

[37] Levy RM, Lamb S, Adams JE. Treatment of chronic pain by deep brain stimulation: long-term follow-up and review of the literature. Neurosurgery 1987; 21: 885-93.

[38] Schuurman PR, Bosch DA, Bossuyt PM, et al. A comparison of continuous thalamic stimulation and thalamotomy for suppression of severe tremor. N Engl J Med 2000; 342: 461-8.

[39] Starr PA, Turner RS, Rau G, et al. Microelectrode-guided implantation of deep brain stimulators into the globus pallidus internus for dystonia: techniques, electrode locations, and outcome. J Neurosurg 2006; 104(4): 488-501.

[40] Videnovic A, Metman LV. Deep brain stimulation for Parkinson's disease : prevalence of adverse events and need for standardized reporting. Mov Disord 2008; 23(3): 343-9.
[41] Chou YC, Lin SZ, Hsieh WA, et al. Surgical and hardware complications in subthalamic nucleus deep brain stimulation. J Clin neurosci 2007; 14(7): 643-9.

[42] Sansur CA, Frysinger RC, Pouratian N, et al. Incidence of symptomatic hemorrhage after stereotactic electrode placement. J Neurosurg 2007; 107(5): 998-1003.

[43] Voges J, Waerzeggers Y, Maarouf M, et al. Deep-brain stimulation: long-term analysis of complications caused by hardware and surgery- experiences from a single centre. J Neurol Neurosurg Psychiatry 2006; 77: 868-72.

[44] Ben-Haim S, Asaad WF, Gale JT, Eskandar EN. Risk factors for hemorrhage during microelectrode-guided deep brain stimulation and the introduction of an improved microelectrode design. Neurosurgery 2009; 64(4): 754-62.

[45] Gorgulho A, De Salles AF, Frighetto L, Behnke E. Incidence of hemorrhage associated with electrophysiological studies performed using macroelectrodes and microelectrodes in functional neurosurgery. J Neurosug 2005; 102(5): 888-96.

[46] Beric A, Kelly PJ, Rezai A, et al. Complications of deep brain stimulation surgery. Stereotact Funct Neurosurg 2001; 77: 73-8.

[47] Binder DK, Rau GM, Starr PA. Risk factors for hemorrhage during microelectrode-guided deep brain stimulator implantation for movement disorders. Neurosurgery 2005; 56(4): 722-32.

[48] Gross RE, Krack P, Rodriguez-Oroz MC, Rezai AR, Benabid AL. Electrophysiological mapping for the implantation of deep brain stimulators for Parkinson's disease and tremor. Mov Disord 2006; 21(Suppl 14): S259-83.

[49] Gervais-Bernard H, Xie-Brustolin J, Mertens P, et al. Bilateral subthalamic nucleus stimulation in advanced Parkinson's disease: five year follow-up. J Neurol 2009; 256(2): 225-33.

[50] Hariz MI. Complications of deep brain stimulation surgery. Mov Disord 2002; 17(Suppl 3): S162-6.

[51] Joint C, Nandi D, Parkin S, Gregory R, Aziz T. Hardware-related problems of deep brain stimulation. Mov Disord 2002; 17(Suppl 3): S175-80

[52] Kondziolka D, Whiting D, Germanwala A, Oh M. Hardwarerelated complications after placement of thalamic deep brain stimulator systems. Stereotact Funct Neurosurg 2002; 79: 228-33.

[53] Lyons K, Koller W, Wiklinson S, Pahwa PR. Surgical and devicerelated events with deep brain stimulation. Neurology 2001; 56: A147.

[54] Sillay KA, Larson PS, Starr PA. Deep brain stimulator hardwarerelated infections: incidence and management in a large series. Neurosurgery 2008; 62(2): 360-7.

[55] Oh My, Abosch A, Kim SH, Lang AE, Lozano AM. Long-term hardware-related complications of deep brain stimulation. Neurosurgery 2002; 50: 1268-7.

[56] Hariz MI, Johansson F. Hardware failure in parkinsonian patients with chronic subthlalamic nucleus stimulation is a medical emergency. Mov Disord 2001; 16: 166-8.

[57] Hamel W, Schrader B, Weinert D, et al. Technical complication in deep brain stimulation. Zentralbl Neurochir 2002; 63: 124-7.

[58] Schwalb JM, Riina HA, Skolnick B, Jaggi JL, Simuni T, Baltuch GH. Revision of deep brain stimulator for tremor. Technical note. J Neurosurg 2001; 94: 1010-12.

[59] Hamani C, Lozano AM. Hardware-related complications of deep brain stimulation: a review of the published literature. Stereotact Funct Neurosurg 2006; 84: 248-51.

[60] Okun MS, Tagliati M, Pourfar M, et al. Management of referred deep brain stimulation failures: a retrospective analysis from 2 movement disorders centers. Arch Neurol 2005; 6298): 1250-5.

[61] Sun DA, Yu h, Tatsas AD, et al. Postmortem analysis following 71 months of deep brain stimulation of the subthalamic nucleus for Parkinson disease. J Neurosurg 2008; 109(2): 325-9.

[62] Voon V, Kubu C, Krack P, et al. Deep brain stimulation : neuropsychological and neuropsychiatric issues. Mov Disord 2006; 21(suppl 14): S305-27.

[63] Witt K, Daniels C, Reiff J, et al. Neuropsychological and psychiatric changes after deep brain stimulation for Parkinson's disease: a randomized, multicentre study. Lancet Neurol 2008; 7(7): 605-13.

[64] Nutt JG, Anderson VC, Peacock JH, Hammerstad JP, Burchiel KJ. DBS and diathermy interaction induces severe CNS damage. Neurology $2001 ; 56: 1384-6$

[65] Henderson JM, Tkach J, Phillips M, Baker K, Shellock FG, Rezai AR. Permanent neurological deficit related to magnetic resonance imaging in a patient with implanted deep brain stimulation elec- 
trodes for Parkinson's disease: case report. Neurosurgery 2005; 57: E1063.

[66] Inci S, Erbengi A, Berker M. Pulmonary embolism in neurosurgical patients. Surg Neurol 1995; 43: 123-9.

[67] Spiegel J, Fuss G, Backens M, et al. Transient dystonia following magnetic resonance imaging in a patient with deep brain stimulation electrodes for the treatment of Parkinson disease: Case report. J Neurosurg 2003; 99: 772-4.
[68] Larson PS, Richardson RM, Starr PA, Martin AJ. Magnetic resonance imaging of implanted deep brain stimulators: experience in a large series. Stereotact Funct Neurosurg 2008; 86(2): 92-100.

[69] Tagliati M, Jankovic J, Pagan F, et al. Safety of MRI in patients with implanted deep brain stimulation devices. Neuroimage 2009; 47(Suppl 2): T53-57.

[70] Kleiner-Fisman G, Herzog J, Fisman DN, et al. Subthalamic nucleus deep brain stimulation: summary and meta-analysis of outcomes. Mov Disord 2006; 21 (Suppl 14): S290-304.

Received: December 25, 2010

Revised: July 27, 2011

Accepted: July 28, 2011

(C) Bakay and Smith; Licensee Bentham Open.

This is an open access article licensed under the terms of the Creative Commons Attribution Non-Commercial License (http://creativecommons.org/licenses/by-nc/3.0/) which permits unrestricted, non-commercial use, distribution and reproduction in any medium, provided the work is properly cited. 